2010

\title{
Viral Gastroenteritis In Charleston, West Virginia, In 2007: From Birth To 99 Years Of Age
}

Carolyn M. Wilhelm

Samantha L. Hanna

Christine A. Welch

Haider Shahid

Linda L. Minnich

See next page for additional authors

Follow this and additional works at: https://researchrepository.wvu.edu/faculty_publications

\section{Digital Commons Citation}

Wilhelm, Carolyn M.; Hanna, Samantha L.; Welch, Christine A.; Shahid, Haider; Minnich, Linda L.; Daly, Shane B.; and Udall, John N., "Viral Gastroenteritis In Charleston, West Virginia, In 2007: From Birth To 99 Years Of Age" (2010). Faculty Scholarship. 729.

https://researchrepository.wvu.edu/faculty_publications/729 
Authors

Carolyn M. Wilhelm, Samantha L. Hanna, Christine A. Welch, Haider Shahid, Linda L. Minnich, Shane B. Daly, and John N. Udall 


\title{
Viral Gastroenteritis in Charleston, West Virginia, in 2007: From Birth to 99 Years of Age
}

\author{
Carolyn M. Wilhelm, MD; Samantha L. Hanna; Christine A. Welch, MS; Haider Shahid; Linda L. Minnich, MS; \\ Shane B. Daly; John N. Udall, Jr, PhD, MD
}

овлестіve. To describe factors associated with a rectal swab or stool sample positive for norovirus, rotavirus, or adenovirus.

DESIGN. Retrospective study.

Setting. Charleston Area Medical Center, a regional academic medical center in Charleston, West Virginia.

METHODs. Rectal swab or stool samples were obtained from patients suspected of having viral gastroenteritis. These samples were sent to the Charleston Area Medical Center virology laboratory for testing in 2007. Viral antigen in rectal swab and stool samples is detected by use of commercially available immunoassay kits for each virus. Data were extracted from the virology laboratory database for the following 1-year time period: January 1, 2007, through December 31, 2007. When necessary, additional information was obtained from electronic administrative data on patients.

RESUlts. There were 2,867 rectal swab and stool samples available for viral testing. Of these samples, 1,261 (44\%) were positive for a virus. Of these positive samples, $972(77 \%)$ were positive for norovirus, $182(14 \%)$ were positive for rotavirus, and 110 (9\%) were positive for adenovirus. The patients in the youngest age group had the highest number of test results positive for all 3 viruses. When the test results for the youngest age group (0-9 years) were compared with those for all the other age groups combined (10-99 years), the proportion of positive cases was highest for the youngest age group $(P<.001)$. There were significant seasonal trends for all 3 viruses. Multivariate analysis of norovirus showed that season, source, sex, and age were significant predictors of a positive test result. Multivariate analysis of rotavirus showed that season and source were significant predictors of a positive test result. Multivariate analysis of adenovirus showed that season and age were significant predictors of a positive test result.

Conclusions. We conclude (1) that these 3 viruses are common causes of gastroenteritis in Charleston, West Virginia; (2) that infants and young children are more likely to test positive for these viruses than are older individuals; (3) that norovirus was the most common cause of gastroenteritis; and (4) that there are seasonal trends for all 3 viruses.

Infect Control Hosp Epidemiol 2010; 31(8):816-821

Viral gastroenteritis is a common cause of illness, and persons with a severe form of it may require hospitalization. The clinical signs and symptoms include fever, diarrhea, nausea, vomiting, and abdominal cramping. Causative viruses are transmitted by the fecal-oral route, and there may be seasonal trends. Three of the more common causes of viral gastroenteritis are norovirus, rotavirus, and adenovirus. Historically, rotavirus has been the most common viral etiology of acute gastroenteritis in children. However, a vaccine (RotaTeq; Merck) directed against rotavirus was introduced in the United States in 2006. ${ }^{1}$ Outbreaks of norovirus-associated gastroenteritis are thought to be more common on cruise ships or in hospitals and/or institutions where overcrowding is a problem..$^{2}$ Adenovirus may not be as important a cause of gastroenteritis as the other 2 viruses.

Routine enzyme immunoassay testing of rectal swab and/ or stool samples obtained from patients suspected of having viral gastroenteritis is performed at the Charleston Area Medical Center (CAMC) in Charleston, West Virginia. The viral gastrointestinal panel tests for norovirus, rotavirus, and adenovirus. To our knowledge, there have been no large, longitudinal studies describing factors associated with positivity for these 3 causes of acute gastroenteritis since the introduction of the rotavirus vaccine. The aim of our retrospective

From the Department of Pediatrics, Robert C. Byrd Health Sciences Center, Charleston Division, West Virginia University (C.M.W., J.N.U.), and the Health Education and Research Institute (S.L.H., C.A.W., H.S., S.B.D.) and the Virology Laboratory (L.L.M.), Charleston Area Medical Center, Charleston, West Virginia.

Received October 26, 2009; accepted February 14, 2010; electronically published June 21, 2010.

(C) 2010 by The Society for Healthcare Epidemiology of America. All rights reserved. 0899-823X/2010/3108-0007\$15.00. DOI: 10.1086/654004 
study was to determine the factors associated with a rectal swab and/or stool sample positive for norovirus, rotavirus, or adenovirus in the Charleston area.

\section{METHODS}

\section{Enzyme Immunoassay}

The virology laboratory at the CAMC receives rectal swab and stool samples obtained from patients suspected of having viral gastroenteritis. Samples are sent from 1 of the 4 CAMC hospitals (Women and Children's Hospital, Memorial Hospital, General Hospital, and Teays Valley Hospital) and from other selected hospitals and physician offices in the Charleston area. Viral antigen in rectal swab and stool samples is detected by use of commercially available immunoassay kits for each virus: the IDEIA Norovirus (Oxoid) assay kit for norovirus, the Premier Rotaclone (Meridian Bioscience) assay kit for rotavirus, and the Premier Adenoclone (Meridian Bioscience) assay kit for adenovirus.

\section{Data Collection}

For our study, data were extracted from the CAMC virology laboratory database for the following 1-year time period: January 1, 2007, through December 31, 2007. When necessary, additional information was obtained from electronic administrative data on patients.

The following variables were recorded: the patient's study number, sex, race, and age; the source of sample (rectal swab or stool sample); the month the sample was collected; and the test result. Patients were grouped into the following age ranges: 0-9 years, $10-19$ years, $20-29$ years, $30-39$ years, $40-$ 49 years, $50-59$ years, $60-69$ years, $70-79$ years, $80-89$ years, and $90-99$ years. Although some individuals were tested multiple times, for the purpose of our study, only the first test result was used in the analysis.

Data were analyzed by use of SAS, version 9.2 (SAS Institute). The $\chi^{2}$ test and logistic regression analysis were used to evaluate the data. A $P$ value of less than .05 was considered to be statistically significant. The study protocol was approved by the CAMC institutional review board.

\section{RES ULTS}

There were 2,867 rectal swab and stool samples submitted to the CAMC virology laboratory in 2007 for enteric viral testing. Of these, 2,663 (93\%) were tested for all 3 viruses, 194 (7\%) were tested for 2 viruses, and $10(0.3 \%)$ were tested for only 1 viral antigen. A total of 8,387 viral antigen tests were performed. Of the 2,867 samples submitted for testing, 1,261 $(44 \%)$ were positive for a viral antigen ( 3 samples were positive for 2 viruses).

\section{Sex and Race}

Of the 8,387 viral antigen tests, 4,414 (53\%) were performed on samples obtained from female patients, and 3,973 (47\%) were performed on samples obtained from male patients. This slight predominance of samples from female patients was noted for each of the viruses tested. Of the 2,676 samples tested for norovirus, $1,421(53 \%)$ were obtained from female patients, and 1,255 (47\%) were obtained from male patients $(P=.11)$. Of the 2,857 samples tested for rotavirus, 1,499 $(52 \%)$ were obtained from female patients, and 1,358 (48\%) were obtained from male patients $(P=.08)$. Of the 2,854 samples tested for adenovirus, 1,494 (52\%) were obtained from female patients, and 1,360 (48\%) were obtained from male patients $(P=.02)$. When race was considered, we found that $91 \%$ of the samples were obtained from white patients, 6\% were obtained from African American patients, and 3\% were obtained from Hispanic patients and patients of other races.

\section{Age Groups}

Samples were obtained from patients who were then categorized by age group (from as young as 1 week of age to as old as 99 years of age). The data were analyzed to determine the number of positive test results for each of the 10 age groups (Figure 1). The age group of $0-9$ years accounted for the largest number of test results positive for norovirus ( $n=533)$, which was $38 \%$ of the samples for that age group. The largest percentage of test results positive for norovirus $(n=12)$ belonged to the age group of $90-99$ years, for which $39 \%$ of the samples tested positive, but this accounted for the lowest group number of cases of viral gastroenteritis. With regard to rotavirus, both the largest number $(n=162)$ and the highest percentage (10\%) of positive test results occurred in the age group of $0-9$ years. When examining adenovirus, we found that the age group of $0-9$ years had the largest number of cases of viral gastroenteritis $(n=82)$, whereas the age group of 20-29 years had the highest percentage (7\%) of positive test results.

\section{Type of Sample}

We found that $43 \%$ of stool samples and $35 \%$ of rectal swab samples were positive for norovirus $(P<.001)$ when the type of sample (rectal swab vs stool sample) was evaluated and when the percentage of positive test results was determined for each type of sample. We found that $7 \%$ of the rectal swab samples and $2 \%$ of the stool samples were positive for rotavirus $(P<.001)$ when the type of sample (rectal swab vs stool sample) was evaluated and when the percentage of positive test results was determined for each type of sample. There was no statistically significant difference between rectal swab samples and stool samples when tested for adenovirus $(P=.271)$.

\section{Seasonality}

To determine whether there were seasonal trends, samples were grouped by virus and month in 2007. The months with 


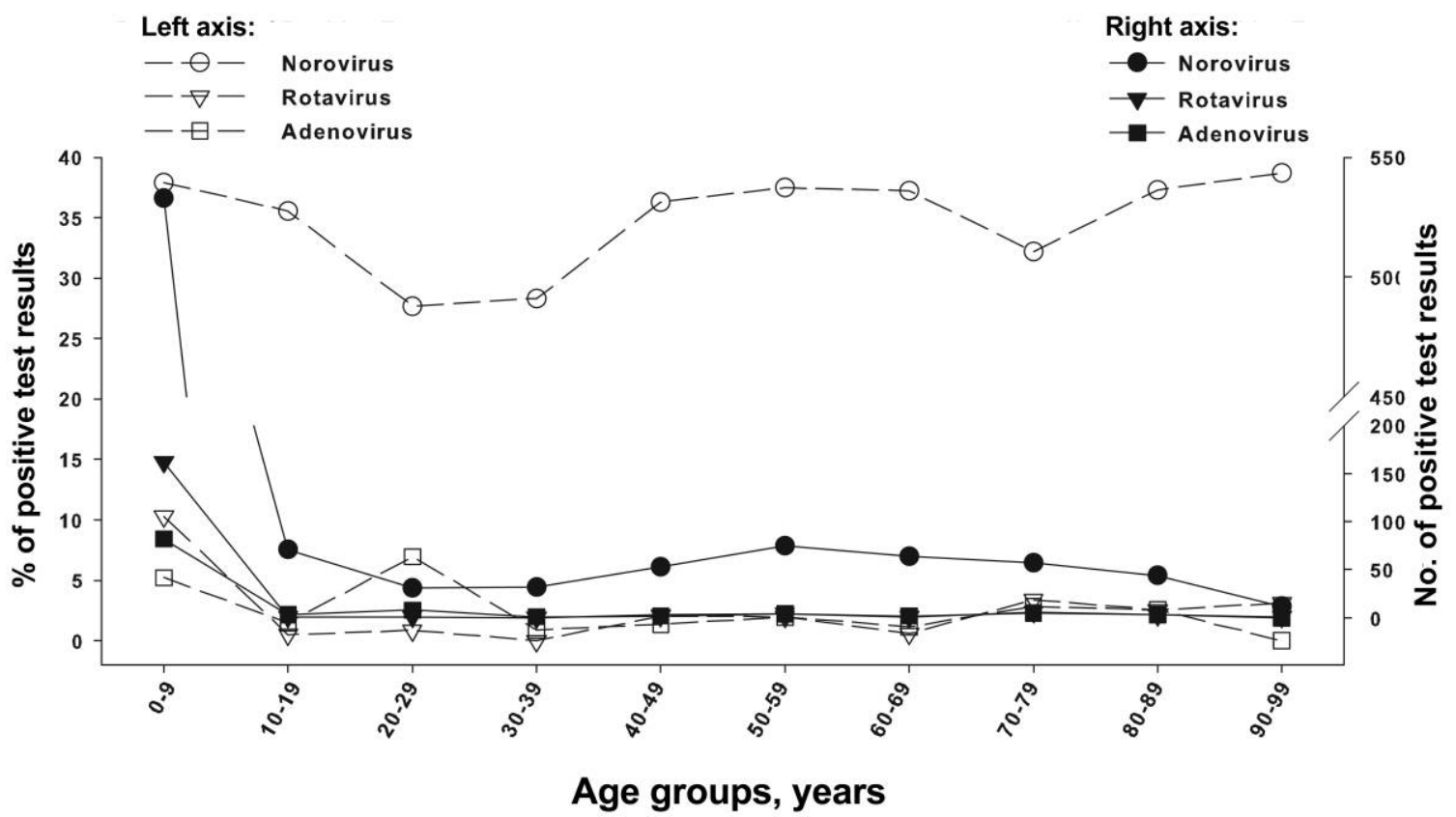

FIGURE 1. Data on test results positive for norovirus, rotavirus, or adenovirus, by age group. The Y-axis is interrupted on the right from 200 to 450 because stool samples were more likely to be positive for norovirus than for the other 2 viruses. The data (from 2007) were extracted from the virology laboratory database of Charleston Area Medical Center in Charleston, West Virginia.

a higher incidence for each virus and the months with a lower incidence for each virus are shown in Figure 2.

With regard to norovirus, significantly more positive test results were observed in January-July than in August-December $(74 \%$ vs $26 \% ; P<.001)$. With regard to rotavirus, significantly more positive test results were observed in January-April than in May-December ( $88 \%$ vs $12 \%$; $P<.001$ ). With regard to adenovirus, significantly more positive test results were observed in October-April than in May-September $(83 \%$ vs $17 \% ; P<.001)$.

\section{Logistic Regression Models}

With 972 test results positive for norovirus and 1,704 test results negative for norovirus, a logistic regression model was developed to predict positivity of the norovirus antigen test. Samples tested during the period from January through July were more likely to be positive than those tested during the period from August through December (odds ratio [OR], 2.53 [95\% confidence interval $\{\mathrm{CI}\}, 2.13-3.01])$. Stool samples were more likely than rectal swab samples to be positive (OR, 1.48 [95\% CI, 1.21-1.82]). Female patients were more likely than male patients to test positive (OR, 1.19 [95\% CI, 1.011.41]). Patients 20-29 years of age were less likely than patients in the other age groups to test positive (OR, 0.62 [95\% CI, 0.41-0.96]), whereas patients 30-39 years of age were less likely than patients in the other age groups to test positive (OR, 0.62 [95\% CI, 0.40-0.95]).

With 182 test results positive for rotavirus and 2,675 test results negative for rotavirus, a logistic regression model was developed to predict positivity of the rotavirus antigen test. Samples tested during the period from January through April were more likely than those tested during the period from May through December to be positive (OR, 11.58 [95\% CI, 7.30-18.38]). Rectal swab samples were more likely than stool samples to be positive (OR, 2.66 [95\% CI, 1.46-4.87]).

With 110 test results positive for adenovirus and 2,744 test results negative for adenovirus, a logistic regression model was developed to predict positivity of the adenovirus antigen test. Samples tested during the period from October through April were more likely than those tested during the period from May through September to be positive (OR, 2.56 [95\% CI, 1.55-4.23]). Patients 0-9 years of age were more likely than patients in the other age groups to test positive (OR, 2.96 [95\% CI, 1.80-4.86]), whereas patients 20-29 years of age were more likely than patients in the other age groups to test positive (OR, 4.28 [95\% CI, 1.83-9.98]).

\section{DISCUSSION}

Acute viral gastroenteritis is a common cause of illness and hospitalization, especially in the pediatric population. ${ }^{3-5}$ One might speculate that transmission among young children may occur more readily, compared with older children and adults, simply because of the nature of children's behavior. However, older individuals may be less likely to seek medical care for evaluation by physicians when they have a bout of "diarrhea."

Racial differences were noted in a study of acute viral gastroenteritis by Fischer et al. ${ }^{5}$ However, the patients in our 


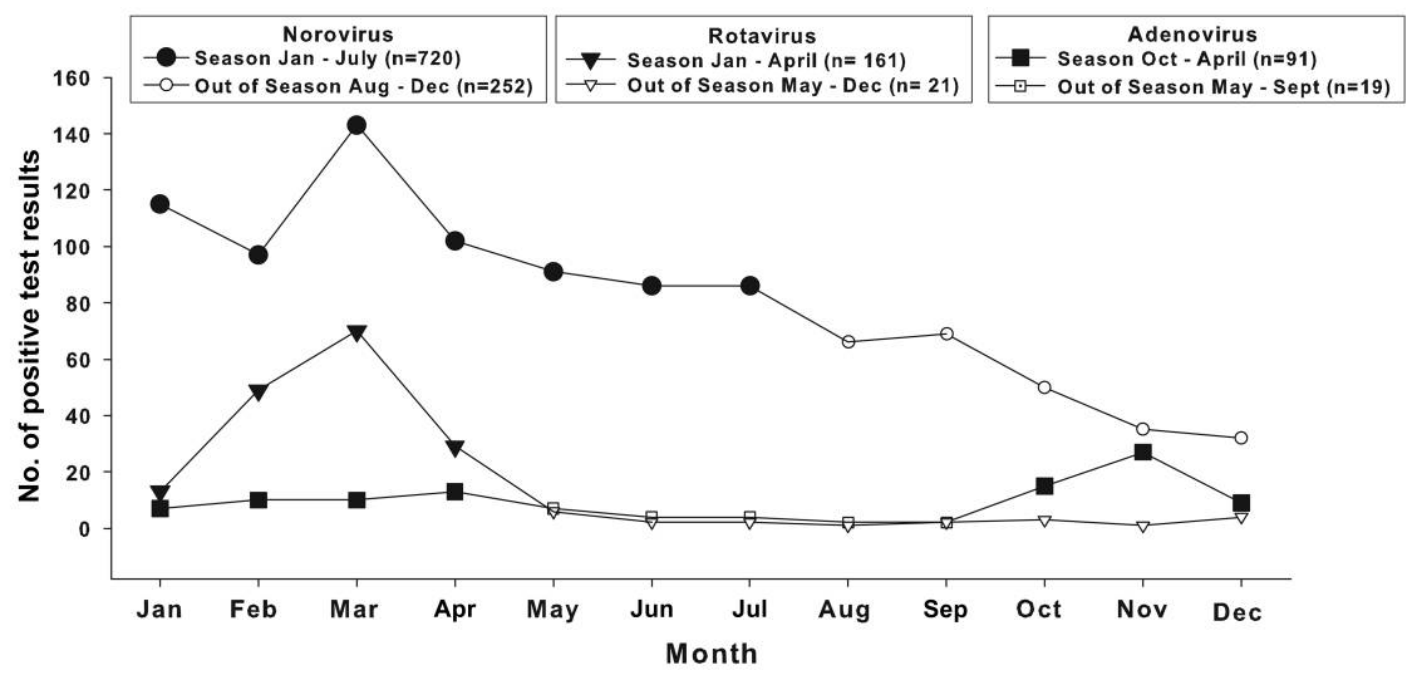

FIGURE 2. Data on test results positive for norovirus, rotavirus, or adenovirus, by month and season. The data (from 2007) were extracted from the virology laboratory database of Charleston Area Medical Center in Charleston, West Virginia.

study paralleled the racial distribution of the Charleston metropolitan area, which is $89 \%$ white, $9 \%$ African American, and $3 \%$ Hispanic and other races. ${ }^{6}$

In an earlier study by our group, ${ }^{7}$ norovirus alone was investigated. The differences between the present study and the previous one are due to the fact that, in the present study, we analyzed (for norovirus, rotavirus, or adenovirus) only the first sample obtained from each patient. In the earlier study, ${ }^{7}$ we examined only the first sample obtained for norovirus. In that study, age groups were assigned differently.

Fischer et $\mathrm{al}^{5}$ demonstrated in their study, which predates the introduction of rotavirus vaccine, that patients with rotavirus infection at hospital admission were more commonly from the younger age groups (ie, 0-9 years). Although we do not know whether our patients were hospitalized or not, our study demonstrates a similar trend: the likelihood of infection due to any of the 3 viruses is highest in the younger age groups. This finding may reflect the immaturity of the immune system in younger children.

Rotavirus has previously been recognized as the most common cause of viral gastroenteritis, ${ }^{1}$ but more recent studies have shown that norovirus may be the more common pathogen in community outbreaks of acute gastroenteritis. ${ }^{8}$ In a 5 -year study undertaken in Australia, ${ }^{9}$ it was found that, in children younger than 5 years of age, the prevalence of calicivirus infection was approximately $9.2 \%$ (ie, detected in 113 of 1,233 samples), with $95 \%$ of strains belonging to norovirus genera. In the study by Wu et al, ${ }^{10}$ stool samples were obtained from children 6-14 years of age with acute gastroenteritis at the Children's Medical Center in Taipei, Taiwan, during the period from January 2004 through March 2006. Of the 201 patients in that study, $44(22 \%)$ tested positive for norovirus; of these 44 patients, 39 (89\%) tested positive for genogroup II norovirus. The authors concluded that norovirus is one of the leading causes of gastroenteritis. ${ }^{10}$ These 2 studies, ${ }^{9,10}$ along with ours, implicate norovirus as an important cause of gastroenteritis in the pediatric population. This may change if a vaccine against norovirus is developed. ${ }^{11,12}$

With the introduction of a vaccine against rotavirus (RotaTeq; Merck) in 2006, studies have attempted to assess the vaccine's effectiveness in decreasing morbidity and mortality in the pediatric population. ${ }^{1}$ The vaccine may account for the reduction in the incidence of rotavirus infection in our study; however, the vaccine does not prevent all cases of rotavirus infection. ${ }^{13,14}$ We do not have data concerning the immunization status of our patients, but our data did show that rotavirus was approximately 4 times more likely to be found in the youngest age group, compared with the older age groups. Positive cases of both norovirus infection and adenovirus infection were also more likely to occur in the youngest age group, compared with the older age groups.

Samples positive for norovirus were more commonly observed during the period from January through July. Samples positive for rotavirus were more commonly observed during the period from January through April. Samples positive for adenovirus were most commonly observed during the period from October through April. Recent reports have confirmed the prevalence of norovirus infection during the winter months. ${ }^{15}$ Other studies have also demonstrated a summertime peak. ${ }^{16}$ Chen et $\mathrm{al}^{4}$ reported peaks for norovirus in the winter through early spring; they also reported that rotavirus infection was most likely to occur during the period from January through March and that adenovirus infection was most likely to occur during the period from October through December. These peaks are similar to the ones we note, but locality, temperature, and elevation above sea level (in addition to other factors) may affect seasonality.

A few other studies have compared the efficacy of rectal 
swab samples with the efficacy of stool samples for the isolation of enteric pathogens. In an early study of staphylococcal colonization in hospitalized burn patients, ${ }^{17}$ rectal swab samples yielded Staphylococcus aureus on culture significantly more often than did simultaneously obtained stool samples $(P<.01)$. Another group of investigators found that the rate of detection of rotavirus in children with acute gastroenteritis was significantly greater with the use of bulk stool samples than with the use of rectal swab samples for an enzyme immunoassay. ${ }^{18}$ To our knowledge, the present study is the first to compare the number of samples positive for norovirus, rotavirus, or adenovirus in rectal swab samples with the number of samples positive for norovirus, rotavirus, or adenovirus in stool samples. Stool samples were more likely than rectal swab samples to be positive for norovirus. We believe that the appropriate technique for obtaining rectal swab samples was not always practiced, and we suggest that stool samples, rather than rectal swab samples, be used to identify norovirus antigen. The Centers for Disease Control and Prevention currently recommends that a large stool sample be used to test for the presence of enteric viruses. ${ }^{19}$

Our study is limited by the fact that it lasted for only 1 year, which makes it difficult to assign seasonality to these viruses. Furthermore, no data were collected concerning clinical findings (eg, data on whether patients were hospitalized or data on the outcome of their conditions). We presume that samples were most likely obtained from individuals with diarrhea or from patients who had other signs and/or symptoms suggestive of gastroenteritis, such as abdominal pain, nausea, and vomiting. In addition, there was no standard procedure for how a sample was obtained and no standard time interval for when a sample was obtained to when it arrived at the laboratory for testing. Finally, each immunoassay kit has a sensitivity and a specificity. Compared with polymerase chain reaction, the IDEIA Norovirus (Oxoid) assay kit has a sensitivity of $72.8 \%$ and a specificity of $100 \%{ }^{20}$ Compared with electron microscopy and a confirmatory blocking enzyme immunoassay ${ }^{21}$ the Premier Rotaclone (Meridian Bioscience) assay kit for rotavirus has a sensitivity of $100 \%$ and a specificity of $92 \% .{ }^{22}$ Compared with a cytopathic effect in tissue culture and electron microscopy, the Premier Adenoclone (Meridian Bioscience) assay kit for adenovirus has a sensitivity of $99 \%$ and a specificity of $100 \%{ }^{23}$ Although the differences in sensitivity and specificity between these 3 assays are not great, they may have influenced the results when we attempted to evaluate the efficacy of rectal swab samples and stool samples for isolating viral antigen.

In conclusion, our data suggest (1) that these 3 viruses are common causes of gastroenteritis in the Charleston area (2) that infants and young children are more likely to test positive for these viruses than are older individuals, (3) that norovirus was the most common cause of gastroenteritis, and (4) that there are seasonal trends for all 3 viruses that are slightly different from those previously reported.

\section{ACKNOWLEDGMENTS}

Financial support. S.L.H. and H.S. received research grants from the CAMC. Potential conflicts of interests. All authors report no conflicts of interest relevant to this article.

Address reprint requests to Christine A. Welch, MS, Health Education and Research Institute, Charleston Area Medical Center, 3200 MacCorkle Avenue, SE, Charleston, WV 25304 (christine.welch@camc.org).

\section{REFERENCES}

1. Committee on Infectious Diseases; American Academy of Pediatrics. Prevention of rotavirus disease: updated guidelines for use of rotavirus vaccine. Pediatrics 2009;123(5):1412-1420.

2. Patel MM, Hall AJ, Vinje J, et al. Norovirus: a comprehensive review. J Clin Virol 2009;44:1-8.

3. Zintz C, Bok K, Parada E, et al. Prevalence and genetic characterization of caliciviruses among children hospitalized for acute gastroenteritis in the United States. Infect Genet Evol 2005;5:281-290.

4. Chen SY, Chang YC, Lee YS, et al. Molecular epidemiology and clinical manifestations of viral gastroenteritis in hospitalized pediatric patients in Northern Taiwan. J Clin Microbiol 2007;45:2054-2057.

5. Fischer TK, Viboud C, Parashar U, et al. Hospitalizations and deaths from diarrhea and rotavirus among children $<5$ years of age in the United States, 1993-2003. J Infect Dis 2007;195:1117-1125.

6. US Census Bureau. State \& county quickfacts. Kanawha County, West Virginia. US Census Bureau Web site. http://quickfacts.census.gov/qfd/ states/54/54039.html. Accessed February 10, 2010.

7. Shahid H, Welch CA, Minnich LL, Daly SB, Wilhelm CM, Udall JN Jr. Gastrointestinal norovirus in the Charleston, West Virginia area-2007: birth to 99 years of age. Pediatr Infect Dis J 2009;28(10):918-920.

8. Gould D. Management of norovirus in the community. Br J Community Nurs 2009;14(3):117, 119-121.

9. Kirkwood CD, Clark R, Bogdanovic-Sakran N, et al. A 5-year study of the prevalence and genetic diversity of human caliciviruses associated with sporadic cases of acute gastroenteritis in young children admitted to the hospital in Melbourne, Australia (1998-2002). J Med Virol 2005;77: 96-101.

10. Wu TC, Liu HH, Chen YJ, Tang RB, Hwang BT, Yuan HC. Comparison of clinical features of childhood norovirus and rotavirus gastroenteritis in Taiwan. J Chin Med Assoc 2008;71(11):566-570.

11. LoBue AD, Thompson JM, Lindesmith L, et al. Alphavirus-adjuvanted norovirus-like particle vaccines: heterologous, humoral, and mucosal immune responses protect against murine norovirus challenge. J Virol 2009; 83:3212-3227.

12. Guo L, Zhou H, Wang M, et al. A recombinant adenovirus prime-viruslike particle boost regimen elicits effective and specific immunities against norovirus in mice. Vaccine 2009;27:5233-5238.

13. Patel MM, Tate JE, Selvarangan R, et al. Routine laboratory testing data for surveillance of rotavirus hospitalizations to evaluate the impact of vaccination. Pediatr Infect Dis J 2007;26:914-919.

14. Tate JE, Panozzo CA, Payne DC, et al. Decline and change in seasonality of US rotavirus activity after the introduction of rotavirus vaccine. $P e$ diatrics 2009;124:465-471.

15. Mounts AW, Ando T, Koopmans M, et al. Cold weather seasonality of gastroenteritis associated with Norwalk-like viruses. J Infect Dis 2000; 181(suppl 2):S284-S287.

16. Marshall JA, Hellard ME, Sinclair MI, et al. Incidence and characteristics of endemic Norwalk-like virus-associated gastroenteritis. J Med Virol 2003;69:568-578.

17. Crossley K, Solliday J. Comparison of rectal swabs and stool cultures for the detection of gastrointestinal carriage of Staphylococcus aureus. J Clin Microbiol 1980;11:433-434.

18. Stockman LJ, Staat MA, Holloway M, et al. Optimum diagnostic assay 
and clinical specimen for routine rotavirus surveillance. J Clin Microbiol 2008;46:1842-1843.

19. Lew JF, LeBaron CW, Glass RI, et al; Centers for Disease Control and Prevention. Recommendations for collection of laboratory specimens associated with outbreaks of gastroenteritis. MMWR Recomm Rep 1990;39(RR-14):1-13.

20. IDEIA Norovirus [package insert]. Denmark House, Angel Drove, Cambridgeshire, UK: Ely (Oxoid).
21. Premier Rotaclone [package insert]. Cincinnati, Ohio: Meridian Bioscience.

22. Premier Adenoclone [package insert]. Cincinnati, Ohio: Meridian Bioscience.

23. Lennette EH, Schmidt NJ. Diagnostic Procedures for Viral, Rickettsial and Chlamydial Infections. 5th ed. Washington, DC: American Public Health Associates, 1979:942-943. 